\title{
Wing Morphometric and DNA Barcoding Analysis of Two Different Public Health Important Anopheles Mosquito Species
}

\author{
Deepika PANDA 1 Ipsita BISWAL ${ }^{2} \quad$ Jaya Kishor SETH ${ }^{3} \quad$ Tapan Kumar BARIK $^{1,4 *}$ \\ 1,2,3,4 Post Graduate Department of Zoology, Berhampur \\ University, Bhanjabihar-760007, Odisha, INDIA \\ ${ }^{4}$ Post Graduate Department of Biotechnology, Berhampur \\ University, Bhanjabihar-760007, Odisha, INDIA \\ e-mails: 'deepika.02104@gmail.com, 2ipsita.biswal@ \\ gmail.com, ${ }^{3 j k s e t h 52 @ g m a i l . c o m, ~}{ }^{4 *}$ tkbarik@rediffmail.com

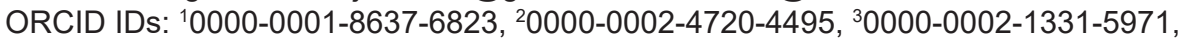 \\ ${ }^{4 *} 0000-0003-3814-4061$
}

\begin{abstract}
Accurate identification and bio-ecology of mosquito vectors are of paramount importance in several respects including the development of species-specific vector control strategies. In the current study, we explored two different advanced tools such as Geometric morphometric (GM) and DNA barcoding analysis, to supplement morpho-taxonomy-based identification of malaria vectors. In landmark-based GM analysis, the pattern of wing shape was examined and we noticed a clear variation in their shape among different Anopheles species. Similarly, in the DNA barcoding studies, the phylogenetic analysis is based upon the marker genes such as mitochondrial Cytochrome oxidase subunit- I ( $\mathrm{mt} \mathrm{COI}$ ), Cytochrome b (mt Cytb), and nuclear D3 domain of 28s rDNA gene sequences were carried out. The Neighbor-joining tree was formed by distinctive conspecific clusters illustrating genetic variation among different groups of Anopheles species. Further, genetic exchange among different Anopheline populations was analyzed by considering the COI gene sequences. In the gene flow study, the frequent genetic divergence, as well as gene exchange among Anopheles stephensi mosquito populations, was observed. Our findings suggested that GM analysis of the wing shape along with the DNA barcoding approach can effectively be used together for accurate identification of mosquito vectors including Anopheles stephensi and Anopheles barbirostris.
\end{abstract}

Key words: Integrated Taxonomic approach, Geometric morphometric, Molecular analysis, Mosquito vector, Anopheles stephensi, Anopheles barbirostris

Panda, D., Biswal, I., Seth J.K., \& Barik, T.K. (2021). Wing morphometric and DNA barcoding analysis of two different public health important Anopheles mosquito species. Journal of the Entomological Research Society, 23(3), 239-255. 


\section{INTRODUCTION}

In India, Malaria, Dengue, Lymphatic filariasis, Japanese encephalitis, Chikungunya, Zika are major vector-borne diseases. Mosquitoes are the well-known vector-borne disease-transmitting agent. There are about 3,583 different Culicidae species reported across the globe (Harbach, 2021). The family Culicidae is a huge and most abundant group which is classified into two different medically important subfamilies such as Anophelinae and Culicinae, among those subfamilies, Anophelinae comprises about 488 valid species. In India, around 58 Anopheles mosquito species have been reported to date (Dev and Sharma 2013). Most Anopheles mosquito species act as a vector for malarial parasites and some other also transmits microfilaria and arboviruses to humans and other animals (Trent, 2005).

For surveillance as well as control of vectors and mosquito-borne diseases, precise identification of mosquito vector species, as well as an understanding of their biology, ecology, and geographical distribution, are crucial (Amini, Hanafi-Bojd, Aghapour, \& Chavshin, 2020). The accurate identification of vector species is essential for developing efficient control strategies. The knowledge of taxonomy has evolved in many different ways, but still, morpho- taxonomy is rated high in mosquito species identification. Traditional mosquito identification has relied on dichotomous keys that explain the physical attributes of a certain life stage. Although this technique has proven to be extremely useful and is still widely used to distinguish many mosquito species, it does have some limitations. It requires taxonomic experts to perform accurate identifications, and it is prone to inappropriate identifications due to the loss of key morphological characters during specimen collection and preservation (Madeira, Duarte, Boinas, \& Costa Osorio, 2021). Further, an integrated taxonomic approach is the need of the hour in order to resolve taxonomic ambiguity, systematics study and phylogenetic analysis in biological sciences (Joshi \& Agarwal, 2021). Keeping in view the importance of the integrated taxonomic approach, during this study we applied two tools i.e. Geometric morphometric and DNA barcoding along with a traditional morpho-taxonomic approach for accurate identification and molecular analysis of mosquito vectors, Anopheles stephensi and Anopheles barbirostris.

The Geometric morphometric (GM) analysis has been introduced as a supplement for error-free identification of species. It has been demonstrated to be an incredible tool for evaluating the correlation between shapes of various organs and structures, including 2D, 3D points representing landmarks (LMs), curves and outlines. It also permits several statistical analyses and allows depicting the shape and size graphically (Lorenz et al, 2017). It became popular because of the current accessibility of modest computational power-specific software (Rohlf, 2003; 2004; 2006; Dujardin, 2010) and digitizing applications suitable even for less experienced users. The advantage of the use of this technique by multivariate regression investigation is that the allometric impact can effortlessly be evacuated from shape analysis, making it conceivable to compare shapes with the least interference from varying sizes (Adams, Rohlf, \& Slice, 2004). Mosquito geometric morphometrics has previously been used to identify 
Wing Morphometric and DNA Barcoding Analysis of Two Different Anopheles mosquito

between genera (Wilke et al, 2016), species within the same genus (Sumruayphol et al, 2016; Chaiphongpachara et al, 2019) and populations within a species (Vidal \& Suesdek, 2012; Morales Vargas et al, 2013). In mosquitoes, wings are the excellent structures generally utilized for morphometric comparisons since it contains veins that encompass natural anatomical landmarks which are ideal for landmarking (Mondal, Devi \& Jauhari 2015).

In recent time, the molecular taxonomic approaches have gained momentum in numerous taxonomic studies on dipterans (Stahls, Vujic, \& Perez-Banon, 2009; Pramual, Wongpakam, \& Adler, 2011) and also employed in identifying several mosquito species (Gonzalez et al, 2010; Laboudi et al, 2011; Ruiz-Lopez et al, 2012). The standard mitochondrial DNA, COI barcode region, has enough information and is very proficient for species identification. It is also helpful in resolving the identification related to ambiguous and cryptic species. In some cases, multiple gene markers have been used to distinguish closely related cryptic as well as sibling species (Lin \& Danforth, 2004). Hence, nuclear gene along with mitochondrial DNA may be used as a supplementary marker to support any conclusions. Moreover, molecular data are broadly utilized for producing molecular phylogenies such as phylogenetic, population genetics, genetic variation and species identification studies. Geographical barriers and climatic differences are the main factors of genetic difference which prevents the geneflow within the same species which gradually leads to speciation. A study on the rate of geneflow gives an idea about the genetic exchange among the different populations of a particular species.

Malaria affects an estimated 219 million people worldwide each year, resulting in over 400,000 deaths (WHO, 2021). India contributes substantially to the global malaria burden with approximately 1,86,532 malaria cases reported in the year 2020 (NVBDCP, 2021). Odisha state of India accounts for about $22 \%$ of malaria cases and $10 \%$ mortality due to malaria alone (NVBDCP, 2021). Out of thirty districts of this state, most of the cases were reported from the Western, Northern and Southern regions of the state (Pradhan et al, 2016). Several mosquito vectors of Anopheles, Culex and Aedes are found in Odisha state and study site in particular. An. culicifacies, An. stephensi, An. minimus mainly act as malaria vectors and $C x$. vishnui, $C x$. tritaeniorhynchus, $C x$. gelidus, Cx. quinquefaciatus, An. barbirostris, An. peditaeniatus, An. subpictus etc acts as vectors of JE in the studied area. Further, there is no sufficient data available on mosquito faunal diversity of southern districts of Odisha state and therefore we have considered this region as our study area.

The primary goal of this study is to identify the public health important Anopheles mosquito species through the geometric-morphometric examination of wing shape variation and by DNA barcoding approach using marker genes such as mitochondrial $\mathrm{COI}$ ( $\mathrm{mt} \mathrm{COI}$ ), Cytochrome $\mathrm{b}$ and nuclear D3 domain of 28s rDNA. In addition, we also analyzed the genetic exchange between different Anopheles mosquitoes based on COI sequences to understand the genetic divergence among Anopheline mosquito species. 
PANDA, D., BISWAL, I., SETH J.K., BARIK, T.K.

\section{MATERIALS AND METHODS}

\section{Mosquito sampling}

A survey of different mosquito breeding habitats was carried out for the updation of mosquito faunal diversity in some southern districts of Odisha state, India (Fig.1). Mosquitoes in their different developmental stages were collected throughout the year by using a variety of standard procedures from multiple locations and transported to the laboratory in preserved condition for identification. Morphological identification of all the field-collected samples was performed using the available identification keys (Christophers, 1933; Barraud, 1934; Tyagi, Munirathinam \& Venkatesh, 2015). After successful identification; the mosquito samples were vouchered and stored for future study.

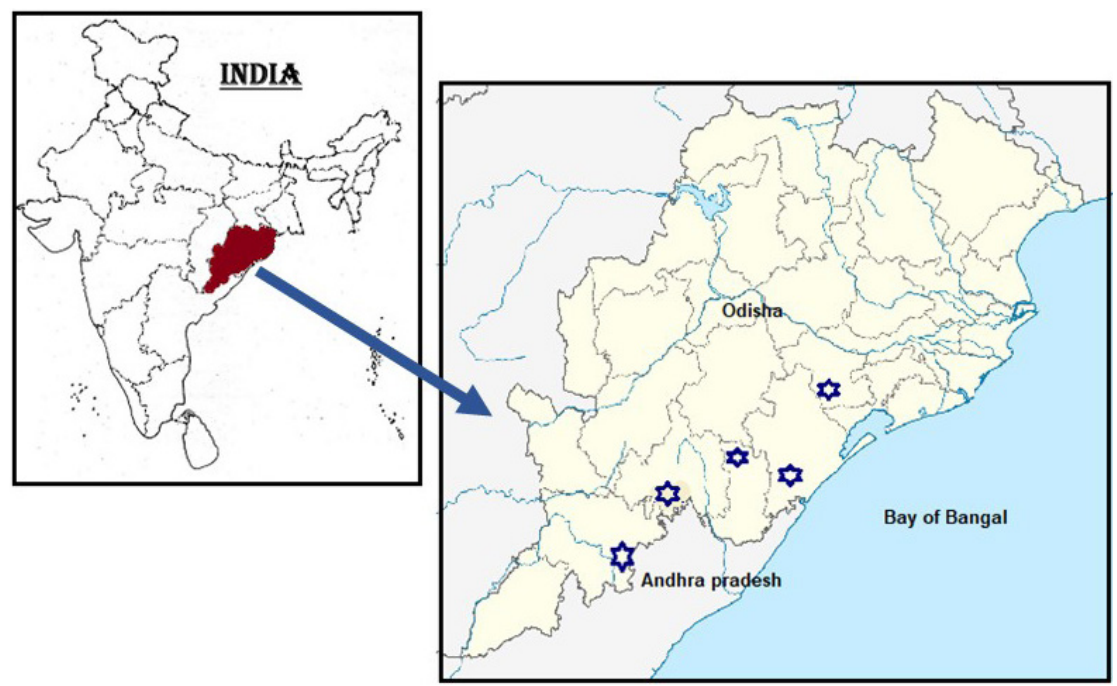

Fig.1. Map showing locations of the mosquito collection sites.

\section{Material preparation for geometric morphometric analysis}

The right-wing of individual adult female mosquito was segregated from the thorax and mounted over a microscope slide with a coverslip. Each wing was then photographed under 40x magnification using Leica DFC320 digital camera coupled with a Leica S6 microscope.

\section{Landmarking for GM analysis}

About 62 photographs of two different mosquito species (Anopheles stephensi $(n=31)$, Anopheles barbirostris $(n=31))$ were chosen and saved as TPS files using the program TPSutil32. The coordinates of 19 landmarks represented through vein intersections were carried out using TPS Dig version 2.31. All the coordinates of landmarks and their descriptions are provided in (Fig.2). In this study, the software Morpho J (Klingenberg, 2011) was used for Principal Component Analysis (PCA) and Discriminate Function Analysis (DFA). 


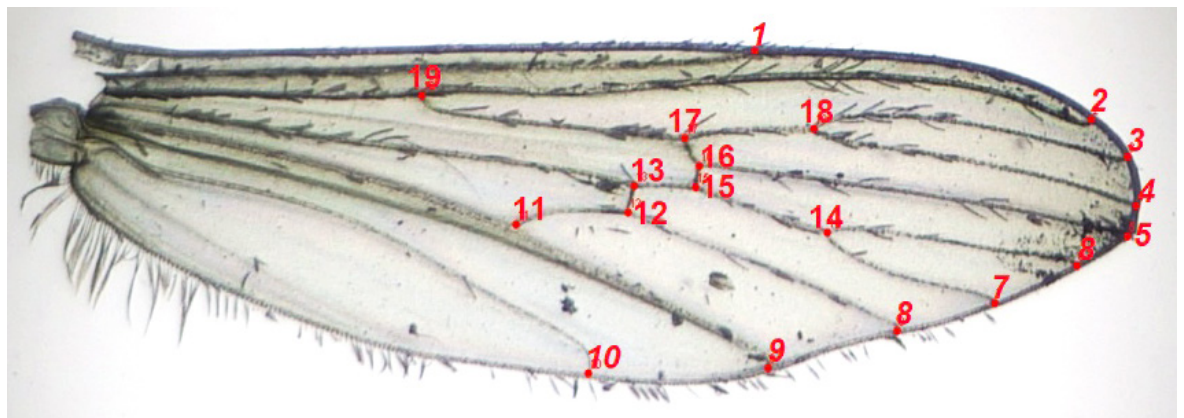

Fig. 2. Landmarks used for PCA and DFA analysis: (1) Intersection of costa(c), (2) Distal end of the radius $(R),(3)$ Radical branch 2, (4) Radical branch 3, (5) Distal end of radius branches 4 and 5, (6) Distal end of M1 and 2, (7) Distal end of M3 and 4, (8) Distal end of cubital vein 1, (9) Distal end of cubital vein 2, (10) Anal vein, (11) Origin of cubital 1, (12) Midpoint branch of cubital 3, (13) Medio-cubital cross vein, (14) Radio-sectoral vein, (15) Midpoint branch Radio medial vein, (16) Radio medial cross vein, (17) Mid-point branch of radial vein, (18) Origin of radius branches 2 and 3, (19) Radial cross vein.

\section{Genomic DNA isolation, PCR amplification and sequencing}

DNA extraction from the whole adult mosquito was carried out as per the Bender Buffer method (Collins et al, 1987) with minor modifications. Further, the isolated DNA was used as a templet for the amplification of mitochondrial COI, Cytb and nuclear 28 s rDNA gene. DNA amplification was carried out using previously described primer pairs by Folmer, Black, Hoeh, Lutz, \& Vrijenhoek 1994; Lyman et al, 1999, Singh et al, 2004 , respectively. The reaction mixture was the same for all the studied genes. All the gene amplification consisting of 1X PCR buffer, $0.5 \mathrm{U}$ Taq DNA, $2.5 \mathrm{mM} \mathrm{MgCl}_{2}, 200 \mu \mathrm{M}$ dNTPs, $10 \mathrm{pmol}$ of each primer, $100 \mathrm{pmol}$ template DNA, total dilution was made up to $25 \mu$ l. The thermal profile for the COI gene consisted of one cycle of $95^{\circ} \mathrm{C}$ for $5 \mathrm{~min}$ followed by 35 cycles of $95^{\circ} \mathrm{C}$ for $30 \mathrm{sec}, 45^{\circ} \mathrm{C}-55^{\circ} \mathrm{C}$ for $30 \mathrm{sec}$ and $72^{\circ} \mathrm{C}$ for $1 \mathrm{~min}$ with a final extension step of $7 \mathrm{~min}$ at $72^{\circ} \mathrm{C}$. The thermal profile for Cytb gene amplification includes an initial denaturation at $94^{\circ} \mathrm{C}$ for $5 \mathrm{~min}$ followed by 35 cycles of denaturation at $94^{\circ} \mathrm{C}$ for $30 \mathrm{sec}$, annealing at $45-47^{\circ} \mathrm{C}$ for 30 seconds, and extension at $72^{\circ} \mathrm{C}$ for $2 \mathrm{~min}$; followed by a final extension of $72^{\circ} \mathrm{C}$ for $7 \mathrm{~min}$. The PCR condition for the $28 \mathrm{~s}$ rDNA gene was similar to COI amplification except for the annealing temperature, which is $48^{\circ} \mathrm{C}$ for $30 \mathrm{sec}$. The amplicons were resolved in $1.5 \%$ agarose gel. Finally, the purified PCR products were outsourced for sequencing. Large no. of individual specimens were sequenced for each species but the only good quality sequence of $\mathrm{COI}$, Cytb and $28 \mathrm{~S}$ rDNA was selected and used in this study for analysis of both the species.

\section{DNA sequence analysis}

The trace files of COI, Cytb and 28s rDNA sequences were edited and assembled using Geneious version 9.0.5 (Biomatters Ltd, Auckland, NZ) (http://www.geneious. com) software and low-quality sequences were excluded at the time of data analysis. The generated nucleotide sequence from each specimen was compared with barcode sequences available on NCBI using nucleotide Basic Local Alignment Search Tool 
(BLASTn), and the final obtained sequences were submitted to NCBI to get the accession number. The accession numbers of the submitted sequences are listed in Table 1. Further, to resolve the genetic relationship among different species, some $\mathrm{mt} \mathrm{COI}$ gene sequences representing the same and related species of Anopheles taxa under study were retrieved from GenBank as replicate data for evaluating the taxonomic position of our target species.

Table 1. Sequence characteristics of COI, Cytb and 28s rDNA (D3).

\begin{tabular}{|c|c|c|c|c|}
\hline \multirow{2}{*}{ Gene } & \multicolumn{2}{|c|}{ Anopheles stephensi } & \multicolumn{2}{c|}{ Anopheles barbirostris } \\
\hline \multirow{2}{*}{ COI } & Sequence length & Accession No. & Sequence length & Accession No. \\
\cline { 2 - 5 } & $668 \mathrm{bp}$ & MN329060 & $615 \mathrm{bp}$ & MN166188 \\
\hline Cytb & $469 \mathrm{bp}$ & MN954649 & $434 \mathrm{bp}$ & MT036262 \\
\hline 28s rDNA & $398 \mathrm{bp}$ & MN319577 & $381 \mathrm{bp}$ & MN318470 \\
\hline
\end{tabular}

\section{Phylogenetic analysis of COI, Cytb and D3 28s rDNA sequences}

Multiple sequence alignment was carried out by the CLUSTAL W algorithm embedded in software package MEGAX (Kumar, Stecher, Li, Knyaz, \& Tamura, 2018) to find out the distance matrix which can be used to make a phylogenetic tree. Further, to study the evolutionary relationships among the mosquito species, the generated mitochondrial $\mathrm{COI}$ and Cytb gene sequences as well as the 28s rDNA gene sequences were subjected to phylogenetic analysis. The phylogenetic analysis of each gene was carried out with a published set of sequences of different mosquitoes for the mapping of the gene phylogenies by Neighbor-joining (NJ) algorithm (Saitou\& Nei, 1987). The evolutionary distances were estimated using the (Kimura 2 Parameter model) $\mathrm{K}_{2} \mathrm{P}$-distance method in MEGAX (Kumar, Stecher, Li, Knyaz, \& Tamura, 2018) with a bootstrap test of 1000 replicates (Felsenstein, 1985). $1^{\text {st }}+2^{\text {nd }}+3^{\text {rd }}+$ noncoding codon positions were included in the study and for each sequence pair, all the ambiguous positions were discarded.

\section{Gene flow estimation}

Estimates of long-term inbreeding effective population sizes and rates of gene flow among regions were made under a Bayesian inference framework using the program DnaSP version 6 (Rozas et al, 2017) separately for different species. Population genetic indices were calculated separately for each mosquito species using nucleotide data of the mt COI gene. Apart from this, the haplotype diversity $(\mathrm{Hd})$, nucleotide diversity $(\mathrm{Pi})$, genetic distances among haplotypes (Fst), number of migration (Nm) were analyzed. Obtained datasets were compared with datasets reported from various regions of India, Pakistan, Sri Lanka, Iran, Thailand, and China.

\section{RESULTS}

Both the immature and mature stages of different types of mosquitoes were collected from different sites of the study area. The immature stages of mosquitoes were reared to the adult stage for morphological identification. The larval habitat from which specimens were obtained includes both natural and artificial container habitats 
Wing Morphometric and DNA Barcoding Analysis of Two Different Anopheles mosquito

whereas adults were collected resting on the various surface from cattle sheds and other human habitats. All the adult mosquitoes were identified morphologically as Anopheles stephensi and Anopheles barbirostris using the available identification keys (Christophers, 1933; Barraud, 1934; Tyagi, Munirathinam \& Venkatesh, 2015).

In the geometric morphometric analysis, the Procrustes sums of squares and the tangent sum of the square were found to be 0.1314 and 0.1310 , respectively. In Principal component (PC) analysis, out of 34 principal components, PC1 and PC2 have the highest eigenvalues and \% variance and thus, these two PCs were chosen for further analysis (Fig.3). The eigenvalues of PC1 and PC2 were found to be 0.0011 and 0.0002 with $\%$ variance $52.091 \%$ and $9.941 \%$ respectively, representing more than $62 \%$ of cumulative variance. The scatter plot of the first two PCs for the 62 specimens showed two distinct clusters thereby the Anopheles barbirostris clustered themselves in the negative axis of PC1 and Anopheles stephensi formed a cluster in the positive axis of PC1 (Fig. $3 \mathrm{C}$ ). The transformation grid of PC1 showed that the lollipop structure of $\operatorname{LM} 7,9,10,11,12,13,14$ and 18 had maximum variation (Fig.3 A). In the PC2 transformation grid, the lollipop of $L M 1,7,8,9,10,14,18$ and 19 showed maximum variation (Fig.3 B). Anopheles stephensi showed a significant shape difference from Anopheles barbirostris which is evident from the Procrustes distance, Mahalanobis distance and T- square value obtained from Discriminant Function Analysis (DFA) having values $0.0642,15.6633,3802.7658$ respectively with $p$-value $<0.0001$.

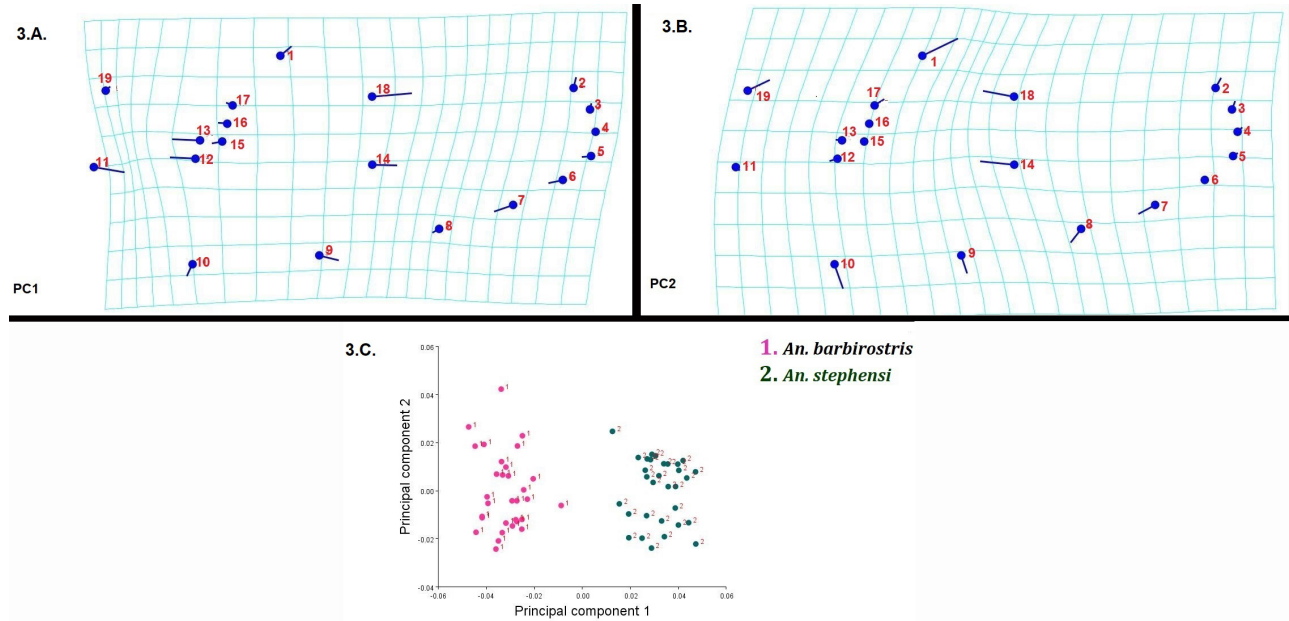

Fig.3. Scatter plot of principal component (PC1 and PC2) and transformation grid representation of variance of 62 samples. Arrows indicate the changes in the relative position of the landmarks.

The COI gene sequences for Anopheles stephensi (668bp) and Anopheles barbirostris $(615 \mathrm{bp})$ were obtained after the final annotation. AT- richness ranges from $67-68 \%$ in these sequences. The Neighbor-joining is conceptually related to the clustering pattern. In this study, the COI gene fragments provide phylogenetic signals and also revealed species boundaries accurately. The optimal NJ tree with the sum of branch length $=0.43167063$ (Fig.4). In the resulted phylogenetic tree, all 
the above-studied specimens form clusters with their respective species. Likewise, the generated Cytb sequences of Anopheles stephensi (469 bp) and Anopheles barbirostris (434bp) were rich in AT content, which is about $74 \%$. A similar NJ tree topology was observed in the case of Cytb geneset, supporting the process of molecular identification (Fig.5). Final annotation obtained from $D_{3}$ gene sequences for Anopheles stephensi (398 bp) and Anopheles barbirostris (381bp) were rich in GC content, which ranges from 55.6\%-57.3\%. Similar to both the mitochondrial genes, these 28s rDNA sequences of the same species cluster together in the phylogeny (Fig.6).

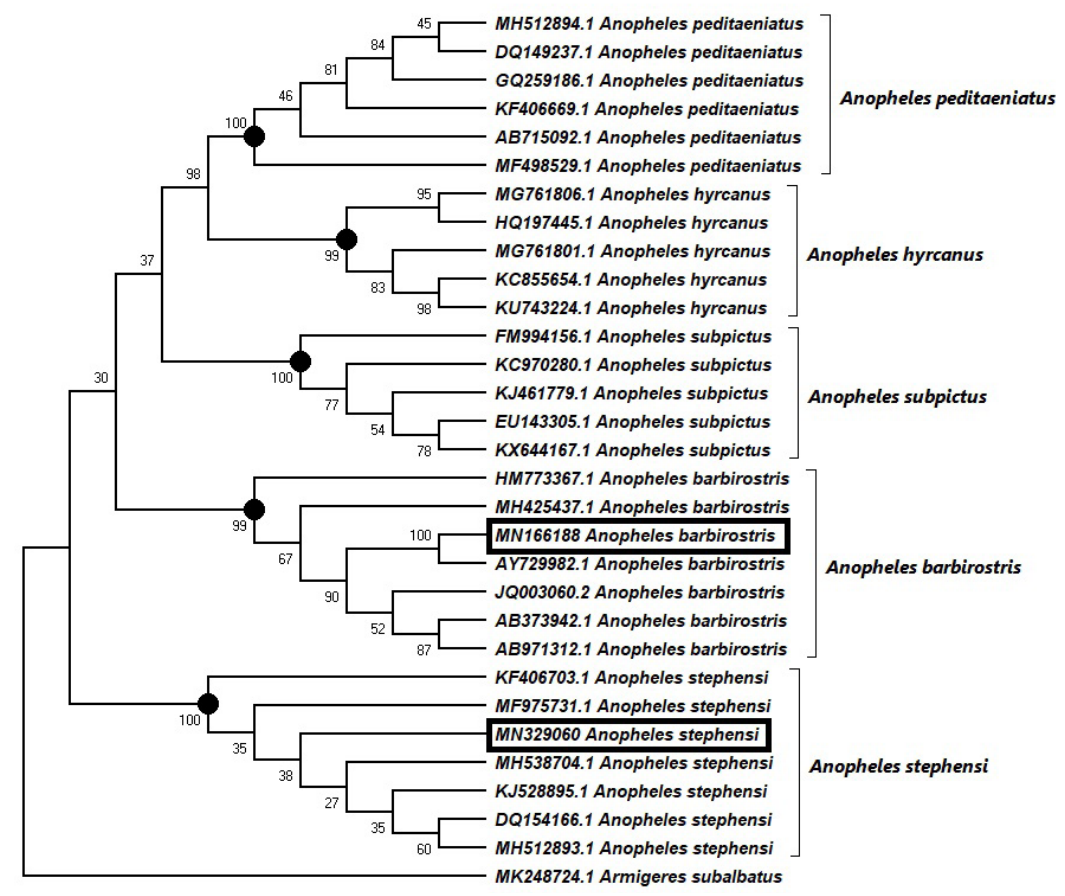

Fig.4. Phylogenetic tree of Anopheles mosquitoes constructed by using the Neighbor-Joining approach. The evolutionary distances have been computed utilizing the $\mathrm{K}_{2} \mathrm{P}$-distance comparisons among $\mathrm{COI}$ sequences and are in the units of the number of base substitutions per site. All the ambiguous positions were removed for each sequence pair. Next to the branches is shown the percentage of replicate trees in which the related taxa clustered together. Species within the box along with the $\mathrm{NCBI}$ accession no. indicates the generated sequence. A sequence of Armigeres subalbatus was used as the out-group.

Haplotype diversity $(\mathrm{Hd})$ and nucleotide diversity $(\pi)$ are two important indicators to measure the diversity of species populations among different geographical strains. Haplotype diversity $(\mathrm{Hd})$ for the $34 \mathrm{COI}$ sequences of Anopheles stephensi was calculated to be 0.631 , an average number of nucleotide differences $(\mathrm{k})$, and nucleotide diversity $(\mathrm{Pi}$ ) was found to be 42.146 and 0.125 , respectively. Genetic differences among the populations of Anopheles stephensi species are provided in Table 2. For Anopheles barbirostris, by considering $41 \mathrm{COI}$ sequences, the haplotype diversity $(\mathrm{Hd})$ was calculated to be 0.897 . An average number of nucleotide differences $(k)$ and nucleotide 
diversity $(\pi)$ was found to be 89.092 and 0.342 , respectively. Genetic differences among the population of $A n$. barbirostris are provided in Table 3. The Nm value between Indian and Pakistan An. stephensi population was greater than 1, which was an indication of frequent genetic exchange between these two places. However, the $\mathrm{Nm}$ value was less than 1 between Indian and other studied populations of An. barbirostris species which indicates a less or no genetic exchange observed among these mosquito populations.

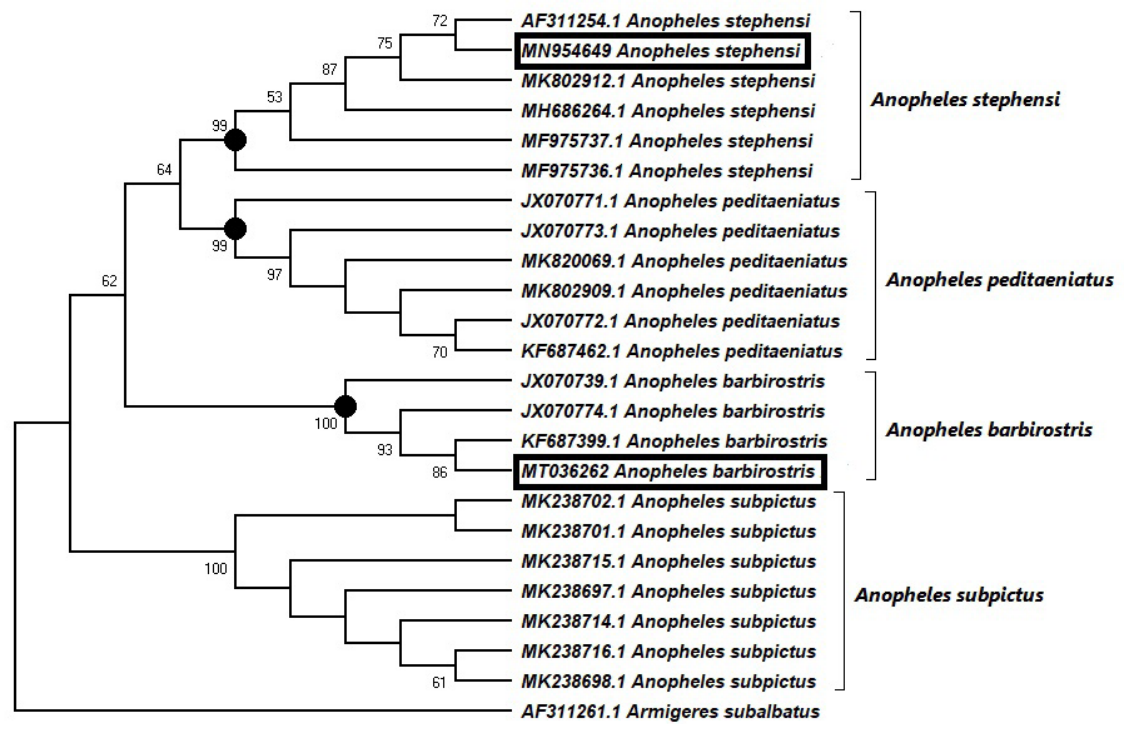

Fig. 5. Phylogenetic tree of Anopheles mosquitoes constructed by using the Neighbor-Joining approach. The evolutionary distances have been computed utilizing the $\mathrm{K}_{2} \mathrm{P}$-distance comparisons among Cytb sequences and are in the units of the number of base substitutions per site. All the ambiguous position were removed for each sequence pair. Next to the branches is shown the percentage of replicate trees in which the related taxa clustered together. Species within the box along with the NCBI accession no. indicates the generated sequence. A sequence of Armigeres subalbatus was used as the out-group.

Table 2. Gene flow properties between different populations of Anopheles stephensi calculated from nucleotide sequence of mtCOI gene. AN1- India, AN2- Pakistan, AN3- Sri Lanka, AN4- Iran. (Hd=Haplotype diversity, $\mathrm{Pi}=$ nucleotide diversity, $\mathrm{Kt}=$ average number of nucleotide difference, $\mathrm{Kxy}=$ inter-population nucleotide differences between the populations, Gst= Genetic differentiation index based on the frequency of haplotypes, Fst= genetic distances among haplotypes, Dxy= average number of nucleotide substitutions per site between all the populations, $\mathrm{Da}=$ average no. of net nucleotide substitutions per site between all the populations, $\mathrm{Nm}=$ number of migration)

\begin{tabular}{|c|c|c|c|c|c|c|c|c|c|c|}
\hline Population 1 & Population 2 & $\mathrm{Hd}$ & $\mathrm{Pi}$ & $\mathrm{Kt}$ & $\mathrm{Kxy}$ & $\mathrm{Gst}$ & $\mathrm{Fst}$ & Dxy & Da & Nm (Based on Fst) \\
\hline AN1 & AN2 & 0.395 & 0.089 & 30.193 & 30.333 & 0.096 & 0.092 & 0.090 & 0.008 & 2.46 \\
\hline AN1 & AN3 & 0.699 & 0.110 & 37.921 & 30.416 & 0.096 & 0.093 & 0.088 & 0.008 & 2.42 \\
\hline AN2 & AN3 & 0.404 & 0.001 & 0.500 & 0.833 & 0.348 & 0.480 & 0.002 & 0.001 & 0.27 \\
\hline AN2 & AN4 & 0.058 & 0.136 & 66.858 & 123.40 & 0.617 & 0.499 & 0.252 & 0.125 & 0.25 \\
\hline AN3 & AN4 & 0.854 & 0.205 & 78.690 & 107.833 & 0.197 & 0.495 & 0.281 & 0.139 & 0.25 \\
\hline AN1 & AN4 & 0.794 & 0.213 & 72.882 & 89.816 & 0.171 & 0.184 & 0.262 & 0.048 & 1.10 \\
\hline
\end{tabular}




$$
\text { PANDA, D., BISWAL, I., SETH J.K., BARIK, T.K. }
$$

Table 3. Gene flow properties between different populations of Anopheles barbirostris calculated from nucleotide sequence of mtCOI gene. P1- Thailand, P2- India, P3-Srilanka, P4- China. (Hd=Haplotype diversity, $\mathrm{Pi}=$ nucleotide diversity, $\mathrm{Kt}=$ average number of nucleotide difference, $\mathrm{Kxy}=$ inter-population nucleotide differences between the populations, Gst= Genetic differentiation index based on the frequency of haplotypes, Fst= genetic distances among haplotypes, Dxy= average number of nucleotide substitutions per site between all the populations, $\mathrm{Da}=$ average no. of net nucleotide substitutions per site between all the populations, $\mathrm{Nm}=$ number of migration)

\begin{tabular}{|c|c|c|c|c|c|c|c|c|c|c|}
\hline Population 1 & Population 2 & $\mathrm{Hd}$ & $\mathrm{Pi}$ & $\mathrm{Kt}$ & $\mathrm{Kxy}$ & $\mathrm{Gst}$ & $\mathrm{Fst}$ & $\mathrm{Dxy}$ & $\begin{array}{c}\mathrm{Nm} \\
\text { Da }\end{array}$ & $\begin{array}{c}\text { (Based } \\
\text { on Fst) }\end{array}$ \\
\hline $\mathrm{P} 1$ & $\mathrm{P} 2$ & 0.839 & 0.198 & 65.930 & 93.433 & 0.169 & 0.539 & 0.281 & 0.151 & 0.21 \\
\hline $\mathrm{P} 2$ & $\mathrm{P} 3$ & 0.757 & 0.234 & 65.990 & 115.175 & 0.281 & 0.849 & 0.409 & 0.348 & 0.04 \\
\hline P2 & $\mathrm{P} 4$ & 0.774 & 0.224 & 77.792 & 125.00 & 0.292 & 0.749 & 0.360 & 0.269 & 0.08 \\
\hline P1 & $\mathrm{P} 4$ & 0.942 & 0.368 & 160.100 & 209.66 & 0.065 & 0.499 & 0.481 & 0.240 & 0.25 \\
\hline P1 & $\mathrm{P} 3$ & 0.923 & 0.191 & 70.456 & 74.466 & 0.096 & 0.153 & 0.202 & 0.031 & 1.38 \\
\hline P3 & P4 & 0.830 & 0.276 & 60.566 & 97.944 & 0.273 & 0.746 & 0.447 & 0.334 & 0.08 \\
\hline
\end{tabular}

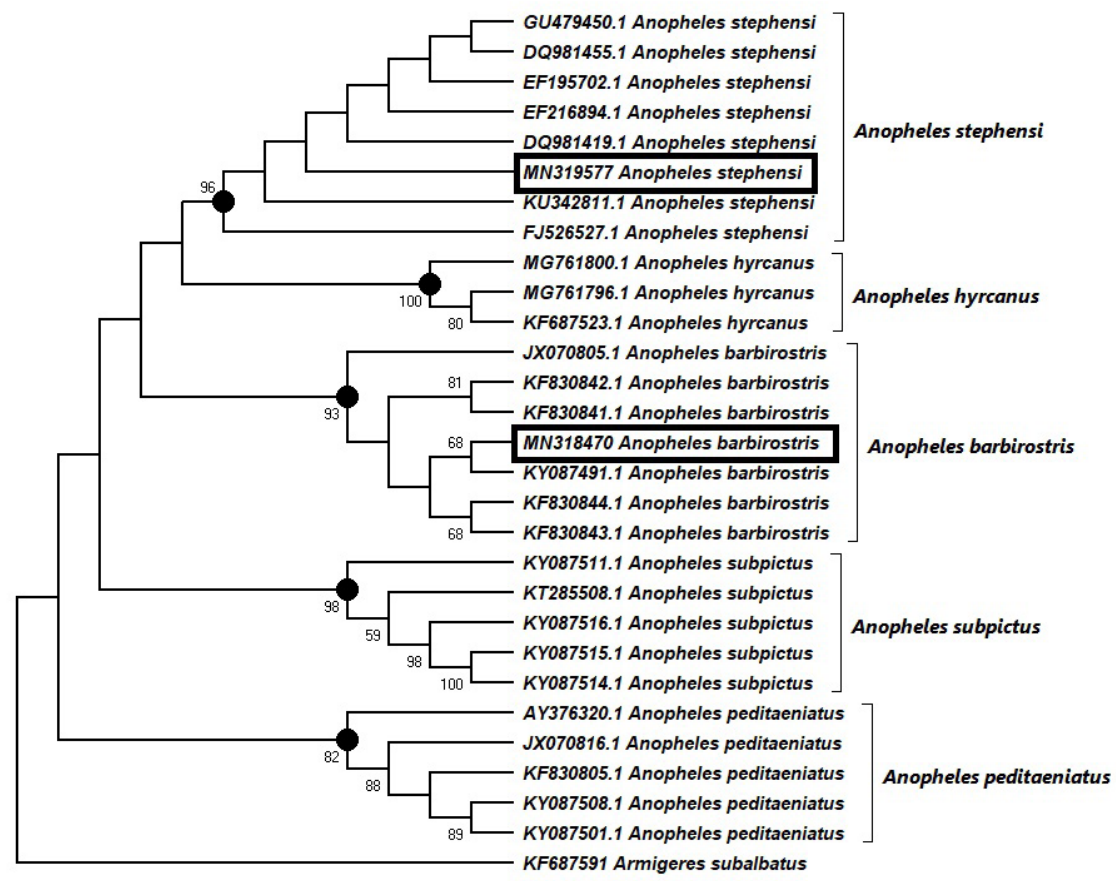

Fig. 6. Phylogenetic tree of Anopheles mosquitoes constructed by using the Neighbor-Joining approach. The evolutionary distances have been computed utilizing the $\mathrm{K}_{2} \mathrm{P}$-distance comparisons among $28 \mathrm{~s}$ rDNA sequences and are in the units of the number of base substitutions per site. All the ambiguous position were removed for each sequence pair. Next to the branches is shown the percentage of replicate trees in which the related taxa clustered together. Species within the box along with the $\mathrm{NCBI}$ accession no. indicates the generated sequence. A sequence of Armigeres subalbatus was used as the out-group. 
Wing Morphometric and DNA Barcoding Analysis of Two Different Anopheles mosquito

\section{DISCUSSION}

Vector-borne diseases account for more than $17 \%$ of all infectious diseases, causing more than 7,00,000 deaths annually (WHO, 2020). The species Anopheles stephensi is a dominant urban vector for both Plasmodium falciparum and Plasmodium vivax, distributed throughout the geographical range of middle-east Indian subcontinent (Nalin et al, 1985; Sharma, 1995; Oshaghi, Yaaghoobi, \& Abaie, 2006). Anopheles barbirostris is also reported as a vector of Japanese encephalitis in India (Tyagi, Munirathinam \& Venkatesh, 2015). Therefore, the unequivocal identification of mosquito specimens is one of the backbones of mosquito and vector surveillance programs and therefore major attention should be given to their genetic diversity study. The combined use of genetic markers provides an idea about the population structure, genetic differentiation and gene flow of species, which is an essential component to design strategies for the management of vector-borne disease (Weeraratne, Surendran, Walton, \& Karunaratne, 2018b).

Even though morpho-taxonomy is regarded as the gold standard method for discrimination of mosquito species, it seems to be quite difficult in the identification of field-collected mosquitoes as they may lose some of their important identifying features during handling. For this reason, there is a need for an alternative technique for identification especially in the case of cryptic and ambiguous species. Various other techniques were also available for mosquito species identification such as cytotaxonomy involving karyotyping of polytene chromosome (Tyagi et al, 2015), isozyme analysis (Knight \& Nayar, 2004), next-generation sequencing approach (NGS) (Muturi, Dunlap, \& Tchouassi, 2021), SNP barcoding with a decision tree algorithm based on machine-learning approach (Swain, Makunin, Dora, \& Barik, 2019), deep learning technique (Mulchandani, Siddiqui, \& Kanani, 2019), convolutional neural networks (CNNs) using image database (Goodwin et al, 2021) etc. Herein, we examined the accuracy of the integrated taxonomic tool using the geometric-morphometrics and DNA barcoding technique.

Geometric morphometric analysis based on quantitative characterization of mosquito wing venation has proved to be a reliable technique for identifying cryptic mosquito species (Morais, Moratore, Suesdek, \& Marrelli, 2010; Lorenz, Marques, Sallum, \& Suesdek, 2012) and also efficiently classify up to species level (Wilke et al, 2016). This was proved to be an effective tool for the rapid, inexpensive and reliable classification of six species of the Aedes genus in France (Martinet et al, 2021). The use of multiple gene markers coupled with landmark-based wing morphometric analysis of insect species including Lepidoptera and Diptera was evidenced from the various studies of Laparie, et al, (2016); Sontigun, et al, (2017); Jin, Hu, Han, \& Chen, (2018). Earlier studies on morphological features of mosquito wings for the identification purpose were also reported by Sumruayphol et al, (2016); Lorenz, et al, (2017); Haarlem, \& Vos, (2018). In the present study, PCA analysis compares two different Anopheline mosquito groups that segregate, forming two separate clusters. LM7, LM9, LM10, LM14 and LM18 showed the highest displacement along the transformation grid of both PC1 and PC2 which specifies that both species are separated from each 
other at these four landmarks. Further, in DFA analysis between two species, the $P$-value is $<0.0001$ which indicates that each group is significantly different from each other with high variation. Thus, our finding indicates that two epidemiologically critical mosquitoes Anopheles stephensi and Anopheles barbirostris were accurately recognized by utilizing wing shape through $\mathrm{GM}$ analysis.

DNA-based methodologies for mosquito identification (Manonmani et al, 2001; Kang, \& Sim, 2013), molecular phylogeny (Shepard, Andreadis, \& Vossbrinck, 2006) and genetic diversity (Pfeiler, Lopez Flores-Lopez, Mada-Velez, Escalante-Verdugo, \& Markow, 2013) have gained increasing adoption in recent years as it is faster to perform and more reliable. Phylogenetic trees are utilized for analysis of gene duplication, estimating rates of diversification, polymorphism, recombination, population dynamics and inferring organismal phylogenies by combining it with other data sources.

Earlier studies have proved the use of the mitochondrial $\mathrm{COI}$ marker in finding more biodiversity and increasing species richness than traditional taxonomic approaches by uncovering undescribed and cryptic species (Hebert, Ratnasingham, \&de Waard, 2003; Schmidt, Schmid-Egger, Morinière, Haszprunar, \& Hebert, 2015; Wilson, Sing, Floyd, \& Hebert, 2017). This COI gene as a molecular marker was used to infer the phylogeny of various dipteran taxa within the genera Aedes, Anopheles and Culex mosquitoes (Ashfaq et al, 2014; Weeraratne, Surendran, \& Parakrama Karunaratne, 2018a; Chan-Chable, Martínez-Arce, Mis-Avila, \& Ortega-Morales, 2019). Recently, a DNA barcode reference library was developed for identifying the mosquito species from Portuguese mosquito fauna, including the most significant vector species (Madeira, Duarte, Boinas, \& Costa Osorio, 2021). In previous reports, rDNA such as ITS ${ }_{2}, \mathrm{D}_{3}$, and Mt DNA COII sequences were also used for differentiation of siblings from five species of Maculatus group (Ma, Li, \& Xu, 2006) collected from China. Similarly, phylogenetic relationships among the Anopheline species was inferred using COI, COII, D3, ITS genes in various other regions of Odisha state of India which proved useful in properly defining species distribution and resolving the ambiguity that normally arises due to morphological taxonomy (Mohanty, Swain, Kar \& Hazra, 2009). Therefore, multiple genes may be required as a supplementary marker to draw any conclusion. Thus, in addition to geometric morphometric analysis of wing shape variation, we employed DNA barcoding of $\mathrm{COI}$, Cytb and $\mathrm{D}_{3}$ domain of $28 \mathrm{~s}$ rDNA sequences for mosquito species identification in the present study. We observed that the composition of generated $\mathrm{COI}$ sequences was AT-rich which is similar to the findings of Cywinska, Hunter, \& Hebert, (2006); Rivera, \& Currie, (2009) on dipterans. In this study, the $\mathrm{mt} \mathrm{COI-based} \mathrm{analysis} \mathrm{strongly} \mathrm{supports} \mathrm{the} \mathrm{positioning} \mathrm{of} \mathrm{Anopheles} \mathrm{stephensi}$ and Anopheles barbirostris by forming distinct clusters with their respective group. Similarly, the phylogenetic repositioning with other two markers such as Cytb and 28s rDNA also supports the cladogenesis of both species. The clustering patterns agreed with the morphological identification, enable distinguishing the individual species based on both the studied gene sequences. Further, in a study, the genetic diversity and evolutionary relationships among An. tessellatus in nine Asian countries at the COI gene were described by Bourke, Wilkerson and Linton in 2021 which revealed 
Wing Morphometric and DNA Barcoding Analysis of Two Different Anopheles mosquito

the exceptional levels of genetic diversity in populations across its known range and identify up to six putative species in the newly determined Tessellatus Complex. The existence of such cryptic diversity has potentially important consequences for vector management and disease control.

Population genetic study illustrated the occurrence of genetic differentiation within the population and the frequent exchange of genes among the population. Fang, et al, (2018) noticed a positive correlation between differences in genetic material among Aedes albopictus population with respect to their geographical distances and concluded that the genetic diversity might occur due to genetic mutation or due to ecological factors which accumulate genetic differences that may lead to reproductive isolation and gradually formation of a new species. We also analyzed the rates of gene flow of two different Anopheles mosquito species in the present study. When the gene flow $\mathrm{Nm}$ value is greater than 1, it means that the gene exchange is frequent, which can prevent the inter-population differentiation caused by genetic drift (Morton, 1977). It may be inferred from this study that due to geographical barriers and differences in climatic conditions between India and other studied regions except for Pakistan, there was a slight difference in $\mathrm{COI}$ gene sequences of $A n$. stephensi. A frequent genetic exchange between Indian and Pakistan populations might be happening due to human activity or fewer physical barriers. However, the $\mathrm{Nm}$ value between different studied populations of $A n$. barbirostris indicates, there might be less or no genetic exchange occurs between Indian with other populations might be due to different environmental and climatic conditions.

In conclusion, although Anopheles stephensi and Anopheles barbirostris are two different species that can be distinguished easily by using identification keys based on morpho-taxonomy. Our study proved here the efficiency of the combined use of wing geometric morphometric analysis and DNA barcoding approach for mosquito identification which can be utilized in the future for the identification of species that are difficult to distinguish. No doubt, only the $\mathrm{COI}$ gene as a molecular marker alone can confirm the species identification along with morpho-taxonomy and geomorphometric analysis but in the present study, we used multiple marker genes to validate the data to strengthen our findings. Furthermore, the result also validated the use of $\mathrm{COI}$ gene sequences for genetic flow analysis that helps to understand genetic diversity among geographically distant populations of different mosquito species.

\section{ACKNOWLEDGMENTS}

The first author would like to thank the Department of Science and Technology, Government of Odisha for financial support.

\section{CONFLICT OF INTEREST}

The authors declare no potential conflict of interest with respect to the research, authorship and/or publication of this article. 


\section{REFERENCES}

Adams, D.C., Rohlf, F.J., \& Slice, D.E. (2004). Geometric morphometrics: ten years of progress following the revolution. Italian Journal of Zoology, 71(1), 5-16.

Amini, M., Hanafi-Bojd, A.A., Aghapour, A.A., \& Chavshin, A.R. (2020). Larval habitats and species diversity of mosquitoes (Diptera: Culicidae) in West Azerbaijan Province, North western Iran. BMC Ecology, 20 (1), 60.

Ashfaq, M., Hebert, P.D., Mirza, J.H., Khan, A.M., Zafar, Y. \& Mirza, M.S. (2014). Analyzing mosquito (Diptera: Culicidae) diversity in Pakistan by DNA barcoding. Plos One, 9, 263 e97268.

Barraud, P.J. (1934). The fauna of British India including Ceylon and Burma. Diptera vol. V. family Culicidae Tribes Megarhinini and Culicini. Taylor \& Francis, London, United Kingdom.

Bourke, B., Wilkerson, R., \& Linton, Y. (2021). Molecular species delimitation reveals high diversity in the mosquito Anopheles tessellatus Theobald, 1901 (Diptera, Culicidae) across its range. Acta Tropica, 215, 105799.

Chaiphongpachara, T., Sriwichai, P., Samung, Y., Ruangsittichai, J., Morales Vargas, R.E., Cui, L., Jetsumon, S., Dujardin, J.P., \& Suchada, S. (2019). Geometric morphometrics approach towards discrimination of three member species of Maculatus group in Thailand. Acta Tropica,192, 66-74.

Chan-Chable, R.J., Martínez-Arce, A., Mis-Avila, P.C., \& Ortega-Morales, A.I. (2019). DNA barcodes and evidence of cryptic diversity of anthropophagous mosquitoes in Quintana Roo, Mexico. Ecology and Evolution, 9, 4692-4705.

Christophers, S.R. (1933). Family Culicidae Tribes Anophelini: The fauna of British India, including Ceylon and Burma- Diptera. Taylor \& Francis, London, 4, 1-271.

Collins, F.H., Mendez, M.A., Rasmussen, M.O., Mehaffey, P.C., Besansky, N.J., \& Finnerty, V. (1987). A Ribosomal RNA gene probe differentiates member species of the Anopheles gambiae complex. American Journal of Tropical Medicine and Hygiene,37, 37-41.

Cywinska, A., Hunter, F.F. \& Hebert, P.D.N. (2006). Identifying Canadian mosquito species through DNA barcodes. Medical \& Veterinary Entomology, 20, 413-424.

Dujardin, J.P., Kaba, D. \& Henry, A.B. (2010). The exchangeability of shape. BMC Research Notes,3 (1), 266.

Fang, Y., Zhang, J., Wu, R., Xue, B., Qian, Q. \& Gao B. (2018). Genetic polymorphism study on Aedes albopictus of different geographical regions based on DNA barcoding. Biomed Research International, 1-10.

Felsenstein, J. (1985). Confidence limits on phylogenies: An approach using the bootstrap. Evolution, 39,783-791.

Folmer, O., Black, M., Hoeh, W., Lutz, R., \& Vrijenhoek R. (1994). DNA primers for amplification of mitochondrial cytochrome $\mathrm{c}$ oxidase subunit I from diverse metazoan invertebrates. Molecular Marine Biology and Biotechnology, 3, 294-299.

Gonzalez, R., Carrejo, N., Wilkerson, R.C., Alarcon, J., Alarcon-Ormasa, J., Ruiz, F., Bhatia, R., Loaiza, J., \& Linton, Y.M. (2010). Confirmation of Anopheles (Anopheles) calderoni Wilkerson, 1991 (Diptera: Culicidae) in Colombia and Ecuador through molecular and morphological correlation with topotypic material. Memorias do Instituto Oswaldo Cruz,105,1001-1009.

Goodwin, A., Padmanabhan, S., Hira, S., Glancey, M., Slinowsky, M, Immidisetti, R., Scavo, L., Brey, J., Sudhakar, B.M.M.S., Ford, T., Heier, C., Linton, Y.M., Pecor, D.B., Quiroga, L.C., \& Acharya, S. (2021). Mosquito species identification using convolutional neural networks with a multitiered ensemble model for novel species detection. Scientific Reports, 11, 13656.

Haarlem, C. \& Vos, R. (2018, Sept 7). Inspecting morphological features of mosquito wings for identification with image recognition tools. doi: http://dx.doi.org/10.1101/410449. Retrieved from https://www. biorxiv.org/content/biorxiv/early/2018/09/07/410449.full.pdf

Harbach, R.E. (2021, March 1). Mosquito taxonomic inventory. Retrieved from http:// mosquito-taxonomicinventory.info. 


\section{Wing Morphometric and DNA Barcoding Analysis of Two Different Anopheles mosquito}

Hebert, P.D., Ratnasingham, S., \& de Waard, J.R. (2003). Barcoding animal life: cytochrome c oxidase subunit 1 divergences among closely related species. Proceedings of the Royal Society of London. Series B, Biological Sciences, 270, 96-99.

Jin, Q., Hu, X., Han, H., \& Chen, F. (2018). A two-step DNA barcoding approach for delimiting moth species: moths of Dongling Mountain (Beijing, China) as a case study. Scientific Reports, 8, 14256.

Joshi, J. \& Agarwal, I. (2021). Integrative taxonomy in the Indian subcontinent: current progress and prospects. Journal of the Indian Institute of Science, 101, 125-149.

Kang, D. \& Sim, C. (2013). Identification of Culex complex species using SNP markers based on high-resolution melting analysis. Molecular Ecology Resources, 13(3), 369-76.

Klingenberg, C.P. (2011). Morphoj: An integrated software package for geometric morphometrics. Molecular Ecology Resources, 11, 353-357.

Knight, J., W., \& Nayar, J., K, (2004). Identification of four common Culex (Culex) (Diptera: Culicidae) species from florida with isozyme analysis. Florida Entomologist, 87(1), 1-5.

Kumar, S., Stecher, G., Li, M., Knyaz, C., \& Tamura, K. (2018). MEGAX: Molecular Evolutionary Genetics Analysis across computing platforms. Molecular Biology and Evolution, 35, 1547-1549.

Laboudi, M., Faraj, C., Sadak, A., Harrat, Z., Boubidi, S.C., Harbach, R.E., Aouad, R.E., \& Linton, Y.M. (2011). DNA barcodes confirm the presence of a single member of the Anopheles maculipennis group in Morocco and Algeria: An. sicaulti is conspecific with An. Iabranchiae. Acta Tropica,118, 6-13.

Laparie, M., Vernon, P., Cozic, Y., Frenot, Y., Renault, D., \& Debat, V. (2016). Wing morphology of the active flyer Calliphoravicina (Diptera: Calliphoridae) during its invasion of a sub-Antarctic archipelago where insect flightlessness is the rule. Biological Journal of the Linnean Society, 119, 179-183.

Lin, C.P. \& Danforth, B.N. (2004). How do insect nuclear and mitochondrial gene substitution patterns differ? Insights from Bayesian analyses of combined datasets. Molecular Phylogeny and Evolution, 30(3), 686-702.

Lorenz, C., Almeida, F., Almeida-Lopes, F., Louise, C., Pereira, S. N., Petersen, V., Vidal, P.O., Virginio, F., \& Suesdek, L. (2017). Geometric morphometrics in mosquitoes: What has been measured? Infection, Genetics and Evolution, 54, 205-215.

Lorenz, C., Marques, T., Sallum, M.A., \& Suesdek, L. (2012). Morphometrical diagnosis of the malaria vectors Anopheles cruzii, An. homunculus and An. bellator. Parasites \& Vectors, 5, 257.

Lyman, D.F., Monteiro, F.A., Escalante, A.S., Cordon-Rosales, C., Wesson, D.M., Dujardin, J., \& Beard, C.B. (1999). Mitochondrial DNA sequence variation among Triatomine vectors of Chargas' disease. American Journal of Tropical Medicine and Hygiene, 60(3), 377-386.

Ma, Y., Li, S. \& Xu, J. (2006). Molecular identification and phylogeny of the Maculatus group of Anopheles mosquitoes (Diptera: Culicidae) based on nuclear and mitochondrial DNA sequences. Acta Tropica, 99, 272-280.

Madeira, S., Duarte, A., Boinas, F., \& Costa Osório, H. (2021). A DNA barcode reference library of Portuguese mosquitoes. Zoonoses and Public Health, 00, 1-11.

Manonmani, A., Townson, H., Adeniran, T., Jambulingam, P., Sahu, S., \& Vijayakumar, T. (2001). rDNA-ITS2 polymerase chain reaction assay for the sibling species of Anopheles fluviatilis. Acta Tropica,78,3-9.

Martinet, J.P., Ferte, H., Sientzoff, P., Krupa, E., Mathieu, B., \& Depaquit, J. (2021). Wing Morphometrics of Aedes Mosquitoes from North-Eastern France. Insects, 12, 341.

Mohanty, A., Swain, S., Kar, S.K, \& Hazra, R.K. (2009). Analysis of the phylogenetic relationship of Anopheles species, subgenus Cellia (Diptera: Culicidae) and using it to define the relationship of morphologically similar species. Infection, Genetics and Evolution, 9, 1204-1224.

Mondal, R., Devi, N.P., \& Jauhari, R.K. (2015). Landmark-based geometric morphometric analysis of wing shape among certain species of Aedes mosquitoes in District Dehradun (Uttarakhand). Journal of Vector Borne Diseases. 52, 122-128. 


\section{PANDA, D., BISWAL, I., SETH J.K., BARIK, T.K.}

Morais, S.A. de, Moratore, C., Suesdek, L., \& Marrelli, M.T. (2010). Genetic-morphometric variation in Culex quinquefasciatus from Brazil and La Plata, Argentina. Memórias do Instituto Oswaldo Cruz,105, 672-676.

Morales Vargas, R.E., Phumala-Morales, N., Tsunoda, T., Apiwathnasorn, C., \& Dujardin, J.P. (2013). The phenetic structure of Aedes albopictus. Infection, Genetics and Evolution, 13, 242-251.

Morton, N.E. (1977). Isolation by distance in human populations. Annals of Human Genetics, 40 (3), 361-365.

Mulchandani, P., Siddiqui, M.U. ,\& Kanani, P. (2019). Real-Time mosquito species identification using Deep learning techniques. International Journal of Engineering and Advanced Technology, 2249-8958.

Muturi, E.J., Dunlap, C., Tchouassi, D.P., \& Swanson, J. (2021). Next generation sequencing approach for simultaneous identification of mosquitoes and their blood- meal hosts. Journal of Vector Ecology, 46(1), 116-121.

Nalin, D.R., Mahood, F., Rathor, H., Muttab, A., Sakai., R., \& Chowdhary, M.A. (1985). A point survey of periurban and urban malaria in Karachi. The Journal of Tropical Medicine and Hygiene, 88, 7-15.

National Vector Borne Disease Control Program (NVBDCP), State wise number of Malaria Cases and Deaths from 2017-2021 (2021, September 23). Retrieved from https://nvbdcp.gov.in/WriteReadData/ 1892s/41082350651617959011.pdf.

Oshaghi, M.A., Yaaghoobi, F., \& Abaie, M.R. (2006). Pattern of mitochondrial DNA variation between and within Anopheles stephensi (Diptera: Culicidae) biological forms suggests extensive gene flow. Acta Tropica, 99, 226-33.

Pfeiler, E., Lopez Flores-Lopez, C.A., Mada-Velez, J.G., Escalante-Verdugo, J. \& Markow, T.A. (2013). Genetic Diversity and Population Genetics of Mosquitoes (Diptera: Culicidae: Culex spp.) from the Sonoran Desert of North America. The Scientific World Journal, 1-11.

Pradhan, A., Anasuya, A., Pradhan, M.M., Kavitha A.K., Kar P., Sahoo K.C., Panigrahi P., \& Dutta A (2016). Trends in Malaria in Odisha, India-an analysis of the 2003-2013 time-series data from the national vector borne disease control program. Plos One, 11(2), e0149126-e0149126.

Pramual, P., Wongpakam, K., \& Adler, P.H. (2011). Cryptic biodiversity and phylogenetic relationships revealed by DNA barcoding of Oriental black flies in the subgenus Gomphostilbia (Diptera: Simuliidae). Genome, 54,1-9.

Rivera, J. \& Currie, D. (2009). Identification of Nearctic black flies using DNA barcodes (Diptera: Simuliidae). Molecular Ecology Resources, 9, 224-236.

Rohlf, F.J. (2003). Tps regr, shape regression, version 1.28. Department of Ecology and Evolution, State University of New York at Stony Brook.

Rohlf, F.J. (2004). Tps dig. department of ecology and evolution state university of New York, Stony Brook, NY.

Rohlf, F.J. (2006). Tps series. Department of Ecology and Evolution, State University of New York, Stony Brook, NY.

Rozas, J., Ferrer-Mata, A., Sánchez-DelBarrio, J.C., Guirao-Rico, S., Librado, P., Ramos-Onsins, S.E., \& Sánchez-Gracia, A. (2017). DnaSP 6: DNA Sequence Polymorphism Analysis of Large Datasets. Molecular Biology Evolution, 34, 3299-3302.

Ruiz-Lopez, F., Wilkerson, R.C., Conn, J.E., McKeon, S.N., Levin, D.M., Quiñones, M.L., Póvoa, M.M., \& Linton, Y.M. (2012). DNA barcoding reveals both known and novel taxa in the Albitarsis Group (Anopheles:Nyssorhynchus) of Neotropical malaria vectors. Parasites \& Vectors, 5, 44.

Saitou, N. \& Nei, M. (1987). The neighbour-joining method: a new method for reconstructing phylogenetic trees. Molecular Biology Evolution, 4, 406-425.

Schmidt, S., Schmid-Egger, C., Morinière, J., Haszprunar, G., \& Hebert, P.D. (2015). DNA barcoding largely supports 250 years of classical taxonomy: identifications for Central European bees (Hymenoptera, Apoidea partim). Molecular Ecology Resources, 15, 985-1000.

Sharma, R.S. (1995). Urban malaria and its vectors Anopheles stephensi and Anopheles culicifacies Diptera: Culicidae) in Gurgaon, India. Southeast Asian Journal of Tropical Medicine and Public Health, 26,172-176. 
Wing Morphometric and DNA Barcoding Analysis of Two Different Anopheles mosquito

Shepard, J.J., Andreadis, T.G., \& Vossbrinck, C.R. (2006). Molecular Phylogeny and Evolutionary Relationships among Mosquitoes (Diptera: Culicidae) from the Northeastern United States Based on Small Subunit Ribosomal DNA (18S rDNA) Sequences. Journal of Medical Entomology, 43(3), 443-454.

Singh, O.P., Chandra, D., Nanda, N., Raghavendra, K., Sunil, S., Sharma, S.K., Dua, V.K., \& Subbarao, S.K. (2004). Differentiation of members of the Anopheles fluviatilis species complex by an Allele-specific polymerase chain reaction based on 28s ribosomal DNA sequences. American Journal of Tropical Medicine and Hygiene, 70(1), 27-32.

Sontigun, N., Sukontason, K.L., Zajac, B.K., Zehner, R., Sukontason, K., Wannasan, A. \& Amendt, J. (2017). Wing morphometrics as a tool in species identification of forensically important blow flies of Thailand. Parasites \& Vectors, 10, 229.

Stahls, G., Vujic, A., \& Perez-Banon, C. (2009). COI barcodes for identification of Merodon hoverflies (Diptera, Syrphidae) of Lesvos Island, Greece.Molecular Ecology Resources,9, 1431-1438.

Sumruayphol, S., Apiwathnasorn, C., Ruangsittichai, J., Sriwichai, P., Attrapadung, S., Samung, Y., \& Dujardin, J.P. (2016). DNA barcoding and wing morphometrics to distinguish three Aedes vectors in Thailand. Acta Tropica, 159, 1-10.

Swain, S.N., Makunin, A., Dora, A.S., \& Barik, T.K. (2019). SNP barcoding based on decision tree algorithm: A new tool for identification of mosquito species with special reference to Anopheles. Acta Tropica, 199, 105152.

Trent, R.J. (2005). Infectious diseases: Molecular medicine. 3rd ed., 193-220. https://doi.org/10.1016/ B978-012699057-7/50008-4

Tyagi, B., Munirathinam, A., \& Venkatesh, A. (2015). A catalogue of Indian mosquitoes. International Journal of Mosquito Research, 50, 50-97.

Tyagi, V., Sharma, A.K., Yadav, R., Sukumaran, D., Agrawal, O.P., \& Veer, V. (2015). Cytotaxonomic examination for identification of Anopheles culicifacies sibling species in central India. Journal of Mosquito Research, 20(5), 1-7.

Vidal, P.O. \& Suesdek, L. (2012). Comparison of wing geometry data and genetic data for assessing the population structure of Aedes aegypti. Infection, Genetics and Evolution, 12, 591-596.

Weeraratne, T.C., Surendran, S.N., \& Karunaratne, S.H.P.P. (2018a). DNA barcoding of morphologically characterized mosquitoes belonging to the subfamily Culicinae from Sri Lanka. Parasites Vectors, 11, 266.

Weeraratne, T.C., Surendran, S.N., Walton, C., \& Karunaratne, S.H.P.P. (2018b). Genetic diversity and population structure of malaria vector mosquitoes Anopheles subpictus, Anopheles peditaeniatus and Anopheles vagus in five districts of Sri Lanka. Malaria Journal,17, 271.

Wilke, A.B.B., de Oliveira Christe, R., Multini, L.C., Vidal, P.O., Wilk-da-Silva, R., d Carvalho, G.C., \& Marrelli, M.T. (2016). Morphometric wing characters as a tool for mosquito identification. Plos One, 11(8), e0161643.

Wilson, J.J., Sing, K.W., Floyd, R.M., \& Hebert P.D. (2017). DNA barcodes and insect biodiversity. In R. G. Foottit, \& P. H. Adler (Eds.). Insect biodiversity: science and society (pp 575-592), Chapter 17, John Wiley \& Sons, United States.

World Health Organization (WHO) (2021, Sept 20). Vector-borne diseases. Retrieved from https://www. who.int/news-room/fact-sheets/detail/vector-borne-diseases.

World Health Organization (WHO) (2021, Sept 20). Retrieved from https://www.who.int/news-room/ fact-sheets/detail/malaria. 